brane dividing two phases is an example of a problem which could be solved by chemical thermodynamics. The chapter on Brownian motion and diffusion will be certainly appreciated by biochemists who wish to under. stand the kinetics of such a phenomenon. Cylindrical and spherical diffusion is discussed in relation to experimental situations. The problems of diffusion in an external field and diffusion in a reacting medium are also solved.

In spite of the fact that this book was obviously written for biochemists, it will certainly be very useful for all people working in laboratories and for scientists concerned with problems of diffusion.

G. Thomaes

\section{SOLVING EQUATIONS}

\section{Approximate Methods for Solution of Differential and Integral Equations}

By S. G. Mikhlin and K. L. Smolitskiy. Translated by Scripta Technica, Inc. Translation edited by Robert E. Kalaba. (Modern Analytic and Computational Methods in Science and Mathematics.) Pp. xi +308. (New York: American Elsevier Publishing Co.; Amsterdam and London: Elsevier Publishing Co., 1967.) 140s.

Is recent decades great advances have been made in devising methods for approximating to the solutions of differential and integral equations; and nowhere has more progress been made than in Russia. The book at hand gives an account of these methods and it is no surprise that emphasis is given to the Russian contributions.

Apart from a short first chapter on the Cauchy problem for ordinary differential equations, the book consists of three further chapters, dealing respectively with numerical methods, analytical methods and methods for solving integral equations. In all cases approximate methods are described. In the numerical case the idea is to cover the domain involved with a grid and to replace the derivatives by the appropriate finite difference approximations based on this grid. This idea is not new, and is in no sense a Russian preserve, as the book may give the impression, and it is perhaps a justified mild criticism that no reference is made to the English work of Southwell and his colleagues of about two decades ago.

There is a brief description of what is called the line method. The basic idea here is to substitute finite differences for the derivatives with respect to one variable, and retain the derivatives relating to other variables. This replaces the equation by a system of differential equations in one fewer variables. The method is intermediate between grid methods and analytical methods and, according to the authors, needs more assessment before it can be considered as established.

There is a large chapter, about one third of the book, on variational methods. These include various analytical methods such as those of least squares, the Ritz method and the Bubnov-Galerkin method. Variational methods in nonlinear problems are discussed. Application is made to a wide variety of problems in classical applied mathematics, but there appears to be no discussion of any problems in the field of fluid dynamics.

The last chapter, on integral equations, covers methods for the usual equations of Fredholm and Volterra. Singular integral equations are also covered.

In a total list of about 140 references there are only about 25 references to work published outside Russia. This one-sidedness seems deplorable in an age when communication should be better, and it is perhaps of little surprise if young mathematicians, and particularly those in Russia, grow up with the erroneous idea that most of the existing knowledge originated in Russia. But this is a criticism of the authors and not of the excellent translation now published by Elsevier.

L. S. GODDARD

\section{BEATING HEARTS}

Factors Influencing Myocardial Contractility

Edited by Ralph D. Tanz, Frederic Kavaler and Jay Roberts. Pp. xxv + 693. (New York: Academic Press; London: Academic Press (London), 1967.) $261 s$.

'I'HIs book collects more than fifty papers given to the Cardiac Muscle Society in August 1966, on various aspects of myocardial behaviour both as a tissue and as singlelayer cell cultures.

Iike many such publications, it makes little conces. sion to non-experts like myself and my numerous colleagues practising cardiac pathology, nor to cardiologists dealing with patients. It is not enough to organize the publication of the papers, which the editors have done admirably. What is lacking is a simple introduction telling how the whole subject has been revolutionized by the electron microscope. This instrument has shown the orderly interdigitating arrays of thick myosin and thin actin filaments (producing the cross striations of myocardium), the transverse and longitudinal tubules which ramify in the sarcoplasm of the muscle cell for its electrochemical and metabolic transport, and the myriads of mitochondria, the large complex granules between the bundles of filaments, which utilize the foodstuffs from the blood to manufacture adenosine triphosphate (ATP). ATP is acted on by ATPase of the myosin filaments, so providing energy for the fibrillar arrays to slide in and out of one another-rather like the bristles of two brushes pressed together and pulled apart again. The whole amazing process depends on the continuous supply of oxygen (since no "debt" can be tolerated), and is profoundly influenced by ions, including calcium, sodium and potassium, and by catecholamines.

Exactly how all these factors influence heart action is the purpose of these papers, most of which I found of absorbing interest. Despite the obvious clinical effects of heart failure, no specific defect has been uncovered; experimentally, it seems that the arrays of thick and thin myofibrils become pulled apart too widely once the limit of the Frank-Starling principle (which states that contraction force is related to previous fibre length) is exceeded.

Several papers deal with monolayer cultures of chick myocardial cells; their beating is influenced by potassium, sodium, barium and serum, but not by calcium, magnesium, noradrenaline, acetylcholine, histamine or curare. The cells can survive at $-190^{\circ} \mathrm{C}$ for three months.

Of current relevance are the studies of the denervated heart, whether by surgery, drugs or by immunological sympathectomy. The myocardium becomes depleted of, and is very sensitive to, catecholamines, which increase excessively its rate and force of contraction. The tonic action of cardiac glycosides is still not understood; some evidence suggests that they make more calcium ions available to the myofibril by inhibiting calcium in the sarcoplasmic reticulum.

Some ingenious apparatus has been designed to measure the thickness and capacity of the heart chambers in living horses and dogs during the cardiac cycle, under various conditions.

There are many papers dealing with the infuence of thyroid, corticosteroids, sex hormones, angiotensin, vasopressin and oxytocin.

Altogether, then, this is a most stimulating and well produced book, which should have a wide sale unless handicapped by its price. I would like to have seen moro courageous and ruthless editorship, to cut out all the repetitious overlapping by so many experts. This would both reduce the book to popular proportions and present a more coherent account of what is known today concern ing the wondrous phenomenon which keeps us alive.

R. E. B. Hudson 